\title{
EL CANTÓN BOLIVIA O CENTRAL DURANTE EL CICLO DE EXPANSIÓN DEL NITRATO ${ }^{1}$
}

\author{
Sergio González
}

\section{* El concepto de Cantón y el origen del CANTÓN BOLIVIA}

\section{Resumen}

Intentaremos establecer la importancia que tuvo el cantón Central o Bolivia (actual II región, Chile) durante el ciclo de expansión del salitre (1880-1930). Para ello se define el concepto de cantón y se establece un nuevo período de expansión (1887-1920). Se concluye que este cantón, a pesar de su relevancia en la historia inicial del salitre durante el s.XIX, perteneció a un distrito salitrero tardío (Antofagasta) y, durante las dos primeras décadas del s. XX, pasó de ser el menos importante de los cinco distritos salitreros (Tarapacá, Tocopilla, Antofagasta, Aguas Blancas y Taltal) a uno de los más significativos.

Palabras claves: cantón - salitre - producción - Chile - Bolivia.

This article establishes the importance of the Central or Bolivia Canton during the nitrate expansion cycle (1880-1930). We define the canton concept and establish a new expansion period (1887-1920). We conclude that the Central Canton, in spite of its relevance in the $19^{\text {th }} \mathrm{Cen}$ tury, belonged to a late saltpeter district, Antofagasta. During the first decades of the $20^{\text {th }}$ Century, it shifted from being the least important of the five saltpeter districts (Tarapacá, Tocopilla, Antofagasta, Aguas Blancas and Taltal) to being one of the most significant.

Key words: canton - nitrate - production - Chile - Bolivia. Recibido: enero 2009. Aceptado: noviembre 2009.
La historia diplomática entre Bolivia y Chile y la historia del salitre se entrecruzan en el cantón Central, ubicado en la región de Antofagasta del Norte Grande de Chile. Las fronteras entre ambos países fueron definidas en el s. XIX, antes del conflicto del Pacífico, por dos tratados internacionales, uno en 1866 y otro en 1874 , en los que se estableció el paralelo $24^{\circ}$ como la frontera internacional. Sin embargo, en el Tratado de 1866, Bolivia y Chile acordaron que se repartirían por mitad el producto de la explotación de los depósitos de guano y de la exportación de los minerales extraídos entre los paralelos $23^{\circ} \mathrm{y}$ $25^{\circ}$ (artículo II). Después, en el Tratado de 1874 , se estableció que "las personas, industrias y capitales chilenos no quedarán sujetos a más contribuciones de cualquiera clase que sean que las que al presente existen" (artículo IV) entre los paralelos $23^{\circ}$ y $24^{\circ}$ (Carrasco 1991). Si bien entonces no era el nitrato el principal producto de interés económico, fue en el marco de estos acuerdos, hacia 1870, cuando se iniciaron las primeras faenas salitreras en el salar del Carmen, ubicado entre los paralelos $23^{\circ}$ y $24^{\circ}$, muy cerca de Antofagasta. Posteriormente, las salitreras se ubicaron en Carmen Alto y Salinas, lo que definió una línea de explotación entre el puerto de Antofagasta y Sierra Gorda (Figura 1).

El concepto de cantón se utilizó desde los inicios del ciclo salitrero en región de Tarapacá, durante las primeras décadas del s. XIX. Se trataba de pampas salitreras donde se instalaban diversas factorías para el beneficio del nitrato, entonces llamadas "paradas". Generalmente, como resultado de estas actividades industriales, emergía en las cercanías algún pueblo que ofrecía los servi-

\footnotetext{
1 Proyecto FONDECYT N ${ }^{0}$ 1080542: "Haciendo visible lo invisible: Asentamientos salitreros en el hinterland del cantón Central, región de Antofagasta (1880-1938)".

2 Instituto de Estudios Internacionales, Universidad Arturo Prat, Casilla 12 1, Iquique, CHILE. Email: pampino@gmail.com
} 
cios y comercio requeridos. Esos pueblos surgieron, a su vez, de las más antiguas paradas salitreras, ya que cuando se agotaba el caliche en el sector, los restos habitacionales de los rudimentarios campamentos iniciales se convertían en asentamientos estables. Así emergió, por ejemplo, el pueblo de La Noria, entre otros.

Posteriormente, con el desarrollo de la industria salitrera a gran escala y la construcción de las diversas líneas de ferrocarril que conectaban el desierto con los puertos y caletas de embarque, las estaciones ferroviarias fueron la base de los nuevos pueblos. Por ello, los cantones salitreros contaban a lo menos con un pueblo central, una estación ferroviaria, un puerto de embarque, una pampa con depósitos de caliche y diversas oficinas que podían pertenecer a una o más compañías. El cantón Bolivia surgió de esta forma, cuando comenzaron a instalarse oficinas salitreras junto a la línea del ferrocarril de Antofagasta a Oruro, como puede apreciarse en la Figura 1.
Durante las diversas etapas de desarrollo del ciclo del nitrato, los nombres de las oficinas salitreras, así como de los cantones, fueron variando. No así los de los puertos y los pueblos, que los conservaron en forma definitiva. Esto es notorio sobre todo en Tarapacá, ya que solamente en la zona salitrera norte de esa provincia, entre los pueblos de Zapiga y Catalina, los nombres de cantones como Zapiga, Matamunqui, Pampa Negra, Pampa Blanca, Chinquiquiray, Sal de Obispo, San Francisco, Dolores, Santa Catalina, se fueron superponiendo, puesto que pertenecieron a períodos diferentes. En Antofagasta, en cambio, los nombres de los cantones tendieron a ser más definitivos desde un comienzo a la vez que abarcaron territorios más amplios (Bermúdez 1963; González 2002).

Como se aprecia en la Figura 2, los cantones salitreros de la actual II región de Chile, fueron El Toco, Bolivia (también Central o El Loa), El Boquete y Aguas

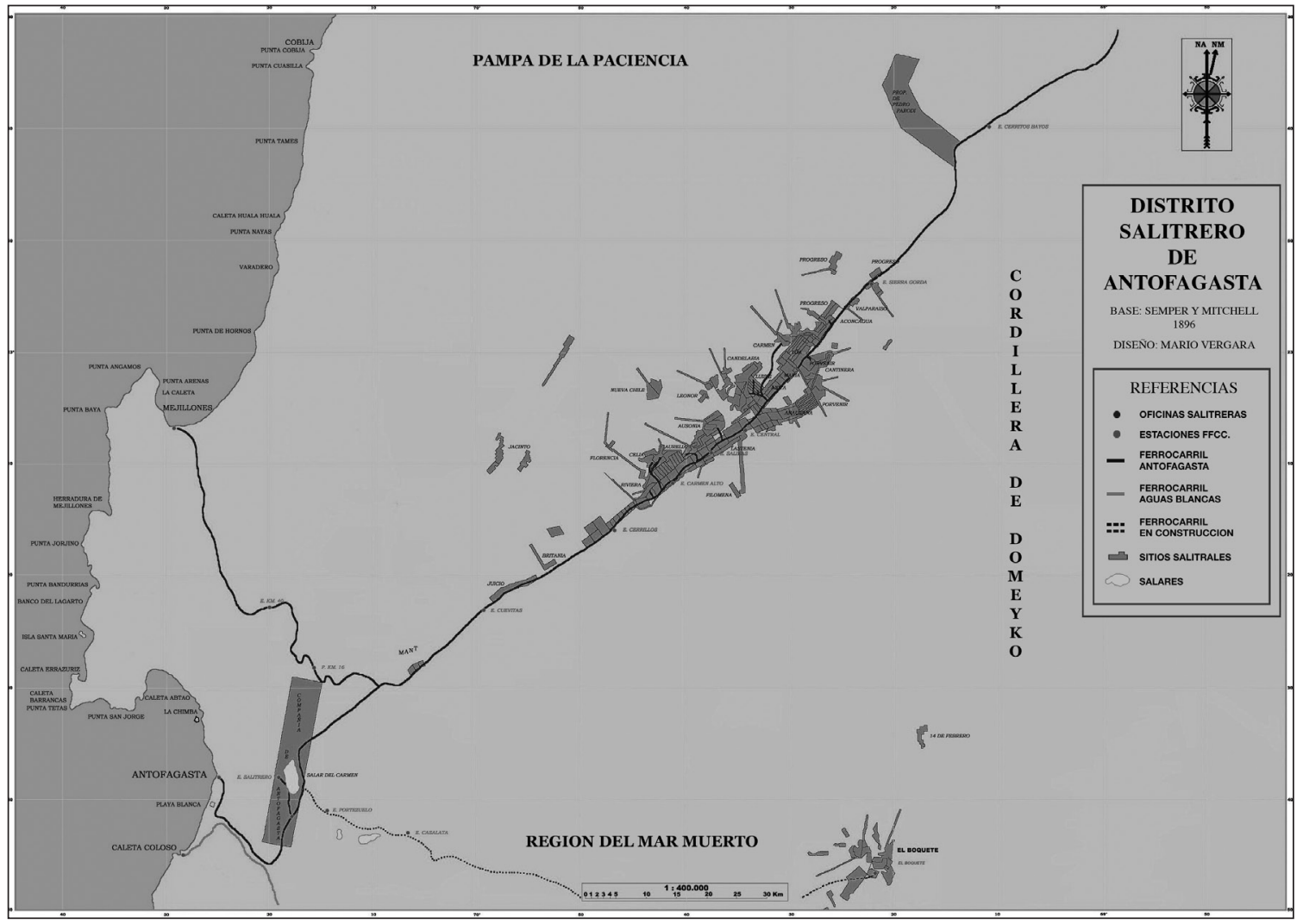

Figura 1. La región de Antofagasta en 1896. Mapa confeccionado por M. Vergara, basado en Semper y Michels (1908). 


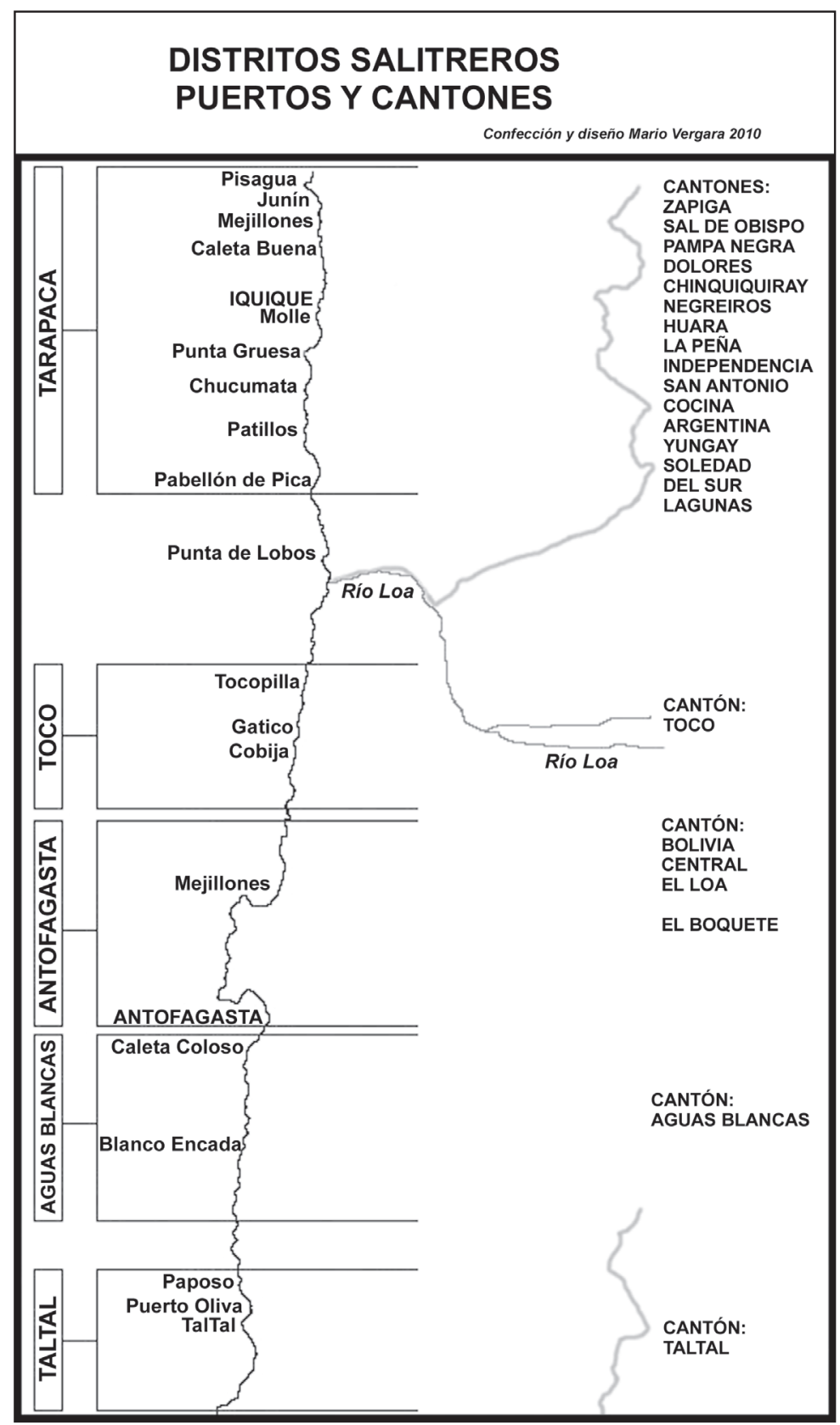

Figura 2. Mapa de los distritos, cantones y puertos salitreros, confeccionado por M. Vergara.

Blancas. Sus puertos de embarque fueron Tocopilla, Mejillones, Antofagasta y Coloso. En las estadísticas de la Asociación Salitrera de Propaganda, que reunía a los productores de nitrato, como veremos más adelante, figuraba cada cantón independiente uno del otro, como si representaran también distritos distintos, con excepción de los cantones El Loa y El Boquete que se incluían en el cantón Bolivia y el cantón Aguas Blancas, respectivamente. Lo mismo acontecía con el cantón de Taltal, que siempre fue considerado como otro distrito. Diferente era la situación de los cantones de Tarapacá que se sumaban todos para representar la producción 


\begin{tabular}{|l|c|c|c|}
\hline \multirow{2}{*}{ Oficinas } & \multicolumn{3}{|c|}{ Desde el $\mathbf{1}^{\circ}$ de abril al 31 de enero } \\
\cline { 2 - 4 } & $1907-08$ & $1906-07$ & $1905-06$ \\
\hline Tarapacá & 18.924 .826 & 21.762 .677 & 21.254 .712 \\
Tocopilla & 4.163 .927 & 3.930 .109 & 4.155 .500 \\
Antofagasta & 4.575 .790 & 3.108 .403 & 2.254 .787 \\
Aguas Blancas & 2.842 .361 & 1.897 .861 & 1.049 .000 \\
Taltal & 3.923 .295 & 3.643 .940 & 3.643 .990 \\
\hline \multicolumn{1}{|c|}{ Total } & 34.430 .199 & 34.342 .990 & 32.357 .989 \\
\hline
\end{tabular}

Tabla 1. Demostración comparada. Salitre elaborado en quintales españoles (Asociación Salitrera de Propaganda. Carlos Otero, Gerente Interino).

total de esta provincia. Para una mejor comprensión de lo señalado, podemos tomar como ejemplo un registro de dicha asociación de productores de nitrato en pleno período de auge.

El concepto Tarapacá, como se registra en la Tabla 1, indica el territorio salitrero entre los cantones Zapiga y Lagunas. Si nos refiriéramos a los puertos de embarque deberíamos decir entre Pisagua y Patillos, pero este último, lamentablemente, nunca se consolidó como puerto de embarque de los cantones correspondientes (Pintados, Buenaventura, Bellavista y Lagunas). Esto se debió a la intervención directa del "Rey del Salitre", John Thomas North, dueño del ferrocarril salitrero, quien lo impidió obligando que la producción de esos cantones saliera por el puerto de Iquique. ${ }^{3}$ En cambio, el concepto Tocopilla hace referencia exclusiva a las salitreras del cantón Toco, que exportaban por el puerto Tocopilla. El concepto Antofagasta, entonces, no se refiere a toda la provincia, sino precisamente a las salitreras del cantón Bolivia o Central, las que exportaban por los puertos de Mejillones y Antofagasta. Por su parte, las salitreras de Aguas Blancas, pertenecían al cantón del mismo nombre más las del cantón El Boquete, siendo Coloso el puerto de embarque (en algunos casos también el puerto de Antofagasta prestaba este servicio). Si tenemos en mente la división político-administrativa actual, la notoria diferencia en producción de salitre elaborado entre Tarapacá y los otros distritos nos lleva a comparar Tarapacá con la sumatoria de la producción de Tocopilla (El Toco), Antofagasta (Bolivia o Central) y Aguas Blancas (Boquete), quedando Taltal como un distrito, cantón y puerto de embarque independiente.

\footnotetext{
3 Para una mejor comprensión del tema ver Blakemore (1977).
}

Los entendidos en la materia consideraban la existencia de cinco distritos salitreros: Tarapacá, El Toco, Antofagasta, Aguas Blancas y Taltal. En algunos casos el distrito y el cantón eran lo mismo, especialmente en aquellos donde las oficinas estaban concentradas en un territorio bien definido. Ejemplo de ello son El Toco y Taltal.

Como se ha dicho, el cantón Bolivia también era conocido como cantón Central o cantón El Loa. Considerando la ubicación del cantón El Toco y los de Aguas Blancas y El Boquete, el concepto de Central tiene sentido para definir al cantón que se ubica justamente en el centro de la provincia de Antofagasta. Como la línea ferrea que definía a este cantón, especialmente en su tramo superior en dirección a Calama sigue el curso del río Loa aunque sin alcanzarlo, podríamos comprender la denominación de cantón El Loa. No obstante el cantón El Toco estaba mucho más asociado geográficamente a este río (ver Figuras 1 y 2).

El cantón Bolivia se vio beneficiado, en su definición como tal, con la temprana construcción de un ferrocarril entre el puerto de Antofagasta y Las Salinas. ${ }^{4}$ Así entonces, desde el salar del Carmen hasta Las Salinas se estructuró una zona de explotación salitrera, donde la Compañía de Salitres y Ferrocarril de Antofagasta, tendría un papel muy significativo en el desarrollo de este distrito. Cabe señalar que un ramal también se internó en el cantón Aguas Blancas, hasta Augusta Victoria.

\footnotetext{
4 La Compañía Exploradora del Desierto de Atacama, principal sociedad que se propuso la explotación de nitrato (y bórax) en el sector del salar del Carmen, recibió el 27 de noviembre de 1873 la autorización del gobierno boliviano para construir un ferrocarril entre el puerto de Antofagasta y la zona de Las Salinas. Ya para entonces la sociedad se denominaba Compañía de Salitres y Ferrocarril de Antofagasta (Thompson y Angerstein 1997:46)
} 


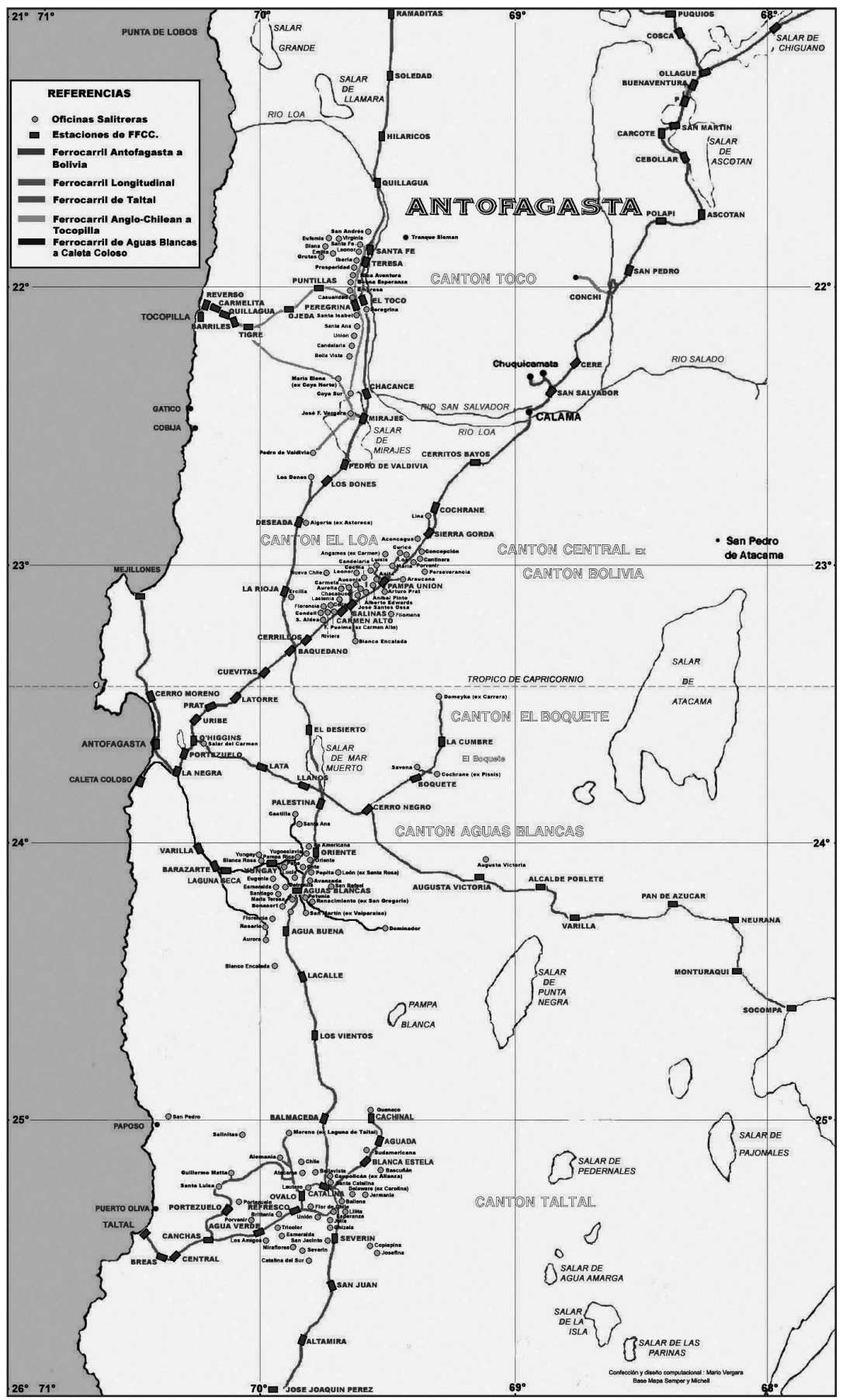

Figura 3. La región de Antofagasta con detalle de cantones y estaciones de ferrocarril. Mapa confeccionado por M. Vergara, basado en Semper y Michels (1908). 


\begin{tabular}{|c|c|c|c|c|}
\hline Oficina & Compañía Salitrera & Agente en Antofagasta & Estación & Puerto de Embarque \\
\hline María & El Loa & Buchanan Jones $\mathrm{C}^{\circ}$ & ídem. & Mejillones \\
\hline Curicó & ídem. & ídem. & ídem. & ídem. \\
\hline Luisis & ídem. & ídem. & ídem. & ídem. \\
\hline Anita & ídem. & ídem. & ídem. & ídem. \\
\hline Cecilia & ídem. & ídem. & ídem. & ídem. \\
\hline Angamos & ídem. & ídem. & ídem. & ídem. \\
\hline Candelaria & ídem. & ídem. & ídem. & ídem. \\
\hline Aconcagua & Lastenia & Baburizza Lukinovic & ídem. & ídem. \\
\hline Filomena & ídem. & ídem. & ídem. & ídem. \\
\hline Araucana & ídem. & ídem. & ídem. & ídem. \\
\hline Perseverancia & ídem. & ídem. & ídem. & ídem. \\
\hline Ausonia & ídem. & ídem. & ídem. & ídem. \\
\hline Celia & ídem. & ídem. & ídem. & ídem. \\
\hline Blanco Encalada & Blanco Encalada & ídem. & ídem. & ídem. \\
\hline Fco. Puelma & Antofagasta & $\mathrm{C}^{\circ}$ Salitrera Antofagasta & ídem. & ídem. \\
\hline A. Edwards & ídem. & ídem. & ídem. & Antofagasta \\
\hline J.S. Ossa & ídem. & ídem. & ídem. & ídem. \\
\hline Anibal Pinto & ídem. & ídem. & ídem. & ídem. \\
\hline Arturo Prat & ídem. & ídem. & ídem. & ídem. \\
\hline C. Condell & ídem. & ídem. & ídem. & ídem. \\
\hline Sargento. Aldea & ídem. & ídem. & ídem. & ídem. \\
\hline J. F. Vergara & ídem. & ídem. & ídem. & ídem. \\
\hline Ercilla & Astoreca $C^{a}$ & Astoreca $C^{a}$ & Unión & Mejillones \\
\hline H. Astoreca & ídem. & ídem. & ídem. & ídem. \\
\hline Lina & J.Sabioncello & Sabioncello Ch & ídem. & Antofagasta \\
\hline
\end{tabular}

Tabla 2. Salitreras del cantón Central o Bolivia que estaban funcionando en 1923 (Ffrench-Davis y Rauld 1923).

El ferrocarril en Antofagasta estuvo tempranamente vinculado a la actividad minera del salitre, a diferencia de Tarapacá en la que, desde las primeras exportaciones de nitrato en 1830 hasta el inicio de la construcción de los primeros ferrocarriles, pasaron más de tres décadas. ${ }^{5} \mathrm{Sin}$ embargo, en diciembre de 1873, cuando todavía era incipiente la exportación salitrera en Antofagasta, la Compañía de Salitres y Ferrocarriles ya había construido la línea

\footnotetext{
5 En 1868 se construyó el ferrocarril de Iquique a La Noria y en
} 1871 el de Pisagua a Negreiros. ferroviaria entre ese puerto y la oficina Salar del Carmen. En 1877 la línea se extendió hasta Carmen Alto, a 127 $\mathrm{km}$ de Antofagasta y, en 1878 había avanzado 1 km hasta Salinas (Blakemore 1996:34).

Después de la Guerra del Pacífico, el ferrocarril ya alcanzaba todo el cantón Central y posteriormente cruzaría los Andes para llegar a Bolivia, transformándose en el único ferrocarril salitrero con una proyección internacional. Respecto a Tarapacá, Blakemore (1977: 58) nos dice que "la línea desde La Noria a la frontera boliviana 
no se comenzó jamás", a pesar de que la empresa Montero Hnos. había recibido una concesión en 1871 del gobierno peruano para construirla.

El largo tramo que recorría el ferrocarril entre el salar del Carmen y Sierra Gorda pudo definir más de un cantón salitrero, sobre todo si consideramos que ese tramo no fue el único en este distrito. Hubo otro que comenzaba en la estación Baquedano y se internaba hacia el oriente hasta Los Dones, pasando por las estaciones Rioja y Deseada, aunque fueron escasas las salitreras que se ubicaron en su vera, como Ercilla y Alcorta o Astoreca (Figura 3).

Si bien no se llamó cantones a los distintos sectores, sí tuvieron denominaciones que sirvieron para ubicarlos con propósitos de comunicación, donde generalmente había estaciones de ferrocarril. Así, tenemos lugares como: Carmen Alto, Salinas, Pampa Central, Pampa Alta, Estación Baquedano, Desvío Buitre, Desvío Santa Rosa, Estación Sierra Gorda, Salinas Ramal, Desvío Peineta, Desvío Maipú, Estación Unión, La Placilla, Paradero La Noria, Paradero Solitario, Zona Longitudinal (Estación Deseada), Paradero Ossa, Paradero Buitre. Estos nombres y otros, fueron cambiando con el tiempo hasta que un pueblo/estación, como Unión, fue concentrando los servicios de todo el cantón Central o Bolivia (Tabla 2).

El cantón Central estuvo directamente asociado a la hazaña de la conquista del desierto salitrero en Antofagasta. Como bien lo ha señalado Bermúdez (1984), el explorador José Antonio Moreno fue quien creyó que había salitre al sur del río Loa, lo que instaría a industriales como Francisco Puelma y José Santos Ossa a iniciar la aventura de buscar nitrato. Precisamente, recién en 1866, en su famosa expedición, José Santos Ossa encontró salitre en las cercanías del salar del Carmen, el que sería considerado como un descubrimiento para Chile. También este cantón estuvo relacionado con el origen de la Guerra del Pacífico y el famoso impuesto "de los 10 centavos" a la Compañía de Salitres y Ferrocarril de Antofagasta, que sería el factor concomitante que desencadenó el conflicto internacional (Ravest 1983).

Lo señalado anteriormente, pone al cantón Central o Bolivia participando de dos momentos fundamentales en la historia del salitre: la exploración del desierto y las causas de la Guerra del Pacífico. Sin embargo, ninguno de ellos será tratado en este artículo, pues aquí se aborda el período de expansión del nitrato, que es posterior a ambos episodios. Es por ello que nos parece fundamental discutir lo que se entiende por ciclo de expansión del nitrato y su relación con el cantón Bolivia.

\section{* El ciclo de expansión del nitrato y EL CANTÓN BOLIVIA}

Tradicionalmente se ha establecido que el ciclo de expansión del salitre se produjo entre 1880 y 1930 , fechas que hasta ahora no han sido discutidas. Sin embargo, si nos preguntamos porqué estas fechas definen ese período, suponemos que la primera (1880) refiere a la ocupación y administración chilena de todos los territorios salitreros desde Pisagua hasta Taltal, y específicamente del de Tarapacá, entonces la principal provincia exportadora de este mineral no metálico. La segunda fecha puede estar referida al inicio de la gran depresión en la que el salitre fue uno de los productos más afectados en el mercado internacional, iniciándose una crisis que no solo fue socialmente muy grave en los pueblos y puertos salitreros, sino que económicamente irreversible. No obstante, ambos argumentos son débiles e incorrectos. Un ciclo de expansión económico no puede estar definido por un factor de orden político o administrativo. Si el argumento fuera que en 1880 ya estaba en operación el sistema de lixiviación Shanks (aquél que permitió el gran salto tecnológico para la expansión productiva del nitrato) podemos afirmar que la introducción de este sistema fue en 1876 y desde entonces en adelante comenzó a implementarse en las nuevas plantas de elaboración. Tampoco 1880 fue un año que haya marcado una inflexión respecto de los años anteriores en capacidad productiva y/o exportación de salitre y yodo. Siguiendo a Hernández (1930: 174), la exportación de nitrato en 1880 fue de 223.974 toneladas métricas. Sin embargo, antes de la Guerra del Pacífico, con excepción del complicado año 1879, la exportación en toneladas métricas fue superior (Tabla 3 ).

Además, los años posteriores a la guerra fueron de altos y bajos. El proceso de regularización de la propiedad de las salitreras de Tarapacá, se convirtió en una gran oportunidad para las oficinas de Antofagasta puesto que les permitió aumentar la producción y exportación de nitrato. Esta situación llegó en gran medida a solucionarse 


\begin{tabular}{|c|c|}
\hline Año & Toneladas Métricas \\
\hline 1876 & 323.642 \\
\hline 1877 & 229.586 \\
\hline 1878 & 323.058 \\
\hline
\end{tabular}

Tabla 3. Exportación de nitrato (1876-1878).

recién el año $1895 \cdot{ }^{6}$ Bermúdez (1984: 158) señala que tanto el cantón Central -a pesar del extraordinario nivel de exportación alcanzado en los años 1879-1881-, como Antofagasta en general, no pudieron competir con los cantones de la antigua provincia peruana debido a sus más altos costos de producción. Efectivamente, los cantones de Antofagasta, Aguas Blancas y Taltal tuvieron un breve auge debido a la Guerra del Pacífico, pero terminado este acontecimiento esa oportunidad se esfumó. De todas formas, Chile aplicó a la exportación de nitrato un impuesto de 0.40 por cada $100 \mathrm{k}$ de salitre, del que quedaron liberadas las salitreras al sur del paralelo $24^{\circ}$ (es decir Aguas Blancas y Taltal) debido a la necesidad de no deprimir esa industria ya instalada. Beneficio, empero, que concluyó definitivamente hacia 1884, generando un gran impacto en esos cantones que además no contaban con un ferrocarril que los uniera con el litoral, a diferencia de Tarapacá, El Toco y Antofagasta.

En el año 1884 se produjo una disminución en la demanda de nitrato en el mercado de Londres, generando, como bien lo señala Bermúdez (1984: 202) "la recesión de las exportaciones salitreras desde comienzos de 1884 [lo que] significó una disminución de un 40 0 50\% de las labores de la pampa". ¿Cómo podríamos calificar de expansión a este período si en 1885 la exportación de nitrato fue de 435.988 toneladas métricas, es decir, no alcanzaba a doblar la exportación de 1880?

\footnotetext{
6 Después de la Guerra del Pacífico, Billinghurst (1886:26) identifica 23 oficinas de máquina, que podríamos calificar de industrias, pues utilizaban el vapor de agua en el proceso de lixiviación, y otras 33 de paradas (algunas de las cuales llegaron después a ser importantes oficinas bajo el sistema Shanks), que fueron canjeadas por certificados o en otras palabras regresadas a sus dueños por parte del Estado chileno. En general éstos no eran los dueños originales sino quienes compraron los certificados que el gobierno peruano había entregado en el proceso de nacionalización del salitre.
}

La situación cambiaría notoriamente cuando comenzó a consolidarse el capital inglés en la producción y exportación del nitrato. Por ejemplo, North organizó en esos años una serie de compañías que marcaron la pauta de lo que sería la industria del salitre en las décadas posteriores. North, en 1883 , en base a los terrenos de la oficina Ramírez, creó The Liverpool Nitrate Company Limited, y en 1885 The Colorado Nitrate Company Ltd., en los terrenos de las paradas Buen Retiro, Peruana y el estacamento Nueva Carolina. $\mathrm{Al}$ año siguiente fundó The Primitiva Company Limited, sobre los terrenos de Primitiva y Abra de Quiroga, para instalar la salitrera Primitiva, la más grande del último tercio del s. XIX. Posteriormente vinieron, entre otras, The New Paccha and Jazpampa Nitrate $C^{0}$, The Lagunas Nitrate $C^{\circ}$ Ltd.y The Lagunas Syndicate Ltd. (Bermúdez 1963).

Semper y Michels (1908: 149), señalan que en 1901 el $55 \%$ de la producción de nitrato lo generaban 48 oficinas inglesas, mientras las chilenas, que eran 11, aportaban un 15\%; otras 12 alemanas un $14 \%$, ocho españolas un $10 \%$ y seis de varias nacionalidades, un $6 \%$.

Según Hernández (1930: 174) a partir de 1887 se hace notoria la expansión de las exportaciones de nitrato (Tabla 4).

\begin{tabular}{|c|c|}
\hline Año & Toneladas Métricas \\
\hline 1887 & 704.244 \\
\hline 1888 & 767.375 \\
\hline 1889 & 947.897 \\
\hline 1890 & 1.063 .277 \\
\hline
\end{tabular}

Tabla 4. Exportación de nitrato (1887-1890).

Sabemos que el año siguiente, 1891, fue un año diferente debido a la guerra civil que envolvió al país y que concluyó con la caída del gobierno y el posterior suicidio del presidente José Manuel Balmaceda. Sin embargo, el salitre ya era el principal producto de exportación del país y su aporte a las arcas fiscales era determinante para la economía nacional. Entre 1891 y 1920, el año de inicio de la primera gran crisis, esos aportes superaron la mitad de los ingresos fiscales del Estado.?

\footnotetext{
7 Los derechos de exportación del salitre y del yodo fueron fijados
} 
Podemos decir, entonces, que el inicio de la expansión del nitrato, en rigor, se aproxima mucho más a 1887 que a 1880 . La pregunta que nos ronda entonces es la fecha de su término.

Quienes han revisado con detalle el desarrollo de la industria del salitre yespecialmente el mercado mundial de los fertilizantes -sobre todo si han seguido los trabajos que en su época realizó el fiscal chileno de propaganda, el ingeniero Alejandro Bertrand- pueden concluir que la crisis del salitre, en tanto fertilizante, comenzó con la Primera Guerra Mundial, pero que, paradójicamente, la economía del salitre fue salvada por la propia guerra al requerir nitrato para uso bélico. Sin embargo, una vez concluida ésta, la crisis se hizo evidente, especialmente por la retirada del mercado alemán del nitrato, ${ }^{8}$ y la posterior competencia con el nitrato sintético.

Si se analiza el período solamente observando la producción y/o la exportación del nitrato se podría concluir que el ciclo continuó expandiéndose con posterioridad a 1920, debido a que esos indicadores seguían aumentando, al igual que la mano de obra y el número de oficinas salitreras.' Sin embargo, es preciso correlacionar esos indicadores con el precio internacional del nitrato, el tipo de cambio (peniques por pesos), los costos de producción y la productividad. De ahí se concluye que la inflexión surge con posterioridad al término de la Primera Guerra Mundial, señalando el inicio de la crisis y el término del ciclo de expansión del nitrato.

En definitiva, 1930 fue la expresión final de una crisis que ya era no solo evidente sino analizada con preocupación por especialistas desde 1920 en adelante, como

por la ley de 23 de diciembre de 1897 , en 3 pesos 38 centavos oro por quintal métrico de salitre y en 1 peso 27 centavos oro por $\mathrm{k}$ de yodo. El fisco percibió entre 1891 y 1912 un monto de 84 millones de libras esterlinas, de las cuales la aduana de Iquique aportó 29.390 .983 y la de Tocopilla 9.933.626. Es decir, Tarapacá aportó prácticamente la mitad de los ingresos fiscales por salitre y yodo en ese período.

8 Antes de la Primera Guerra Mundial Chile producía 392 mil toneladas anuales de nitrógeno. Una vez concluida la guerra "Alemania comenzó a transformar anualmente 112 mil toneladas de nitrógeno amoniacal en nítrico, o sea una cantidad sensiblemente igual al nitrógeno nítrico que antes adquiría en Chile" ( Muraour 1922:328).

9 Para una estadística detallada de estos indicadores ver apéndice estadístico de Cariola y Sunkel (1991). lo podemos observar en la extraordinaria revista Caliche editada por el Instituto Científico e Industrial del Salitre, cuyo director era Belisario Díaz Ossa. Su primer número fue editado en abril de 1919. El interés por la tecnología del nitrato provino en definitiva del deseo de resolver el problema de la crisis económica. Uno de los artículos de esta publicación (correspondiente al $n^{0} 7$ de octubre de 1919), denominado "La ciencia y el porvenir de la industria salitrera" fue escrito originalmente en 1918 y publicado en El Mercurio de Valparaíso. Su autor, Hobsbawn, señala en una de sus partes:

"Por primera vez en su existencia la industria salitrera tiene que afrontar una formidable competencia y carece de toda protección para defenderse. Considerando los enormes intereses nacionales afectados, la culpabilidad solo puede atribuirse al Gobierno del país, debido a la falta absoluta de previsión al no tomar medidas para el estudio profundo de esta gran industria nacional, permitiendo que la explotación se hiciera a su gusto, sin preocuparse de las contingencias del futuro..." (Hobsbawn 1919:263).

Otros autores mencionan distintos factores para explicar la crisis que surge con el fin de la guerra, como la salida del mercado alemán para el nitrato chileno y la alta tasa de impuesto fiscal a las exportaciones (Vidal 1953); la especulación en el mercado mundial del salitre (Reyes 1985); la escasa inversión en propaganda salitrera y la existencia de las combinaciones (Bertrand 1910).

La crisis salitrera fue estudiada en sus más diversas dimensiones durante la llamada Semana del Salitre, realizada en Santiago en 1926, organizada por la Academia de Ciencias Económicas, y contó con la presencia de las más altas autoridades del país, incluido el presidente de la República, Emiliano Figueroa. Notorias figuras del país, políticos, diplomáticos, empresarios salitreros, intelectuales, científicos, entre otros, entregaron su aporte para superar la crisis que ya era evidente (Academia de Ciencias Económicas 1926) Era demasiado tarde, nada evitaría el colapso de 1930.

En síntesis, podemos definir el período de expansión entre 1887 y 1920 . Será entonces en este lapso donde observaremos la relevancia del cantón Central o Bolivia en el marco general del salitre en Chile (Tabla 5). 


\begin{tabular}{|c|c|c|}
\hline Números & Nombre de las oficinas & $\begin{array}{l}\text { Salitre en quintales } \\
\text { españoles }\end{array}$ \\
\hline & Tarapaca & \\
\hline 1 & Amelia $\ldots \ldots \ldots$ & 152.152 \\
\hline 2 & 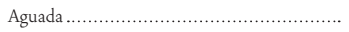 & 294.671 \\
\hline 3 & 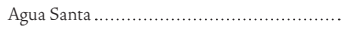 & 444.250 \\
\hline 4 & 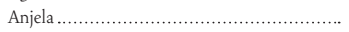 & 142.983 \\
\hline 5 & Aragón & 135.166 \\
\hline 6 & $\ldots+\ldots \ldots \ldots \ldots \ldots \ldots \ldots \ldots \ldots$ & 1.184 .040 \\
\hline 7 & Argentina... & 17.020 \\
\hline 8 & $\ldots \ldots \ldots \ldots \ldots \ldots \ldots \ldots \ldots \ldots \ldots \ldots \ldots . .$. & 98.204 \\
\hline 9 & Buen Retiro........ & 130.981 \\
\hline 10 & Cala Cala & 465.494 \\
\hline 11 & Cataluña ......... & 95.048 \\
\hline 12 & Constancia & 395.600 \\
\hline 13 & Carolina $\ldots \ldots \ldots \ldots \ldots \ldots$ & 149.730 \\
\hline 14 & Cholita ......... & 147.430 \\
\hline 15 & Democracia & 187.543 \\
\hline 16 & Esmeralda...... & 18.860 \\
\hline 17 & Huáscar .............. & 132.953 \\
\hline 18 & Josefina ............. & 96.890 \\
\hline 19 & Kerima .................. & 79.018 \\
\hline 20 & La Palma........ & 262.200 \\
\hline 21 & La Granja.... & 634.800 \\
\hline 22 & Mercedes & 58.603 \\
\hline 23 & North Lagunas ............................................. & 592.675 \\
\hline 24 & 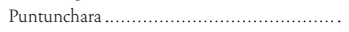 & 289.340 \\
\hline 25 & 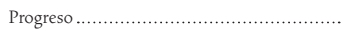 & 43.036 \\
\hline 26 & Providencia $\ldots \ldots \ldots \ldots$ & 124.950 \\
\hline 27 & Ramirez & 572.680 \\
\hline 28 & Reducto ........... & 115.056 \\
\hline 29 & Rosario de Huara ..................................... & 435.380 \\
\hline 30 & Rosario de Negreiros................... & 169.161 \\
\hline 31 & San Pedro & 115.071 \\
\hline 32 & San Antonio ...................... & 108.100 \\
\hline 33 & 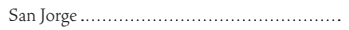 & 366.160 \\
\hline 34 & San Patricio ............................. & 94.372 \\
\hline 35 & San Francisco... & 59.140 \\
\hline 36 & South Lagunas... & 463.680 \\
\hline 37 & Santiago ....................... & 449.650 \\
\hline 38 & Sacramento de $Z \ldots \ldots \ldots \ldots \ldots \ldots$ & 84.540 \\
\hline 39 & Santa Rita ........................ & 43.166 \\
\hline 40 & Santa Lucía....... & 243.340 \\
\hline 41 & Santa Rosa de Huara.... & 192.777 \\
\hline 42 & San Esteban... & 125.704 \\
\hline 43 & San Manuel & 147.414 \\
\hline 44 & 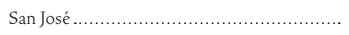 & 150.190 \\
\hline 45 & Tres Marias & 344.061 \\
\hline 46 & Unión ........... & 215.510 \\
\hline 47 & Virginia .......... & 216.342 \\
\hline 48 & Valparaíso ............................. & 658.500 \\
\hline \multirow{2}{*}{49} & Vis .............. & 15.193 \\
\hline & Antofagasta & \\
\hline \multirow[t]{2}{*}{50} & Antofagasta... & 266.280 \\
\hline & Tocopilla & \\
\hline 51 & Buena Esperanza.... & 353.468 \\
\hline 52 & Peregrina $\ldots . . . . . . . . . . . .$. & 294.124 \\
\hline 53 & Santa Isabel del Toco............................. & 239.885 \\
\hline 54 & Santa Fé de del Toco... & 260.682 \\
\hline \multirow{2}{*}{55} & Iberia de Saez ............. & 338.480 \\
\hline & Taltal & \\
\hline 56 & Atacama ........... & 115.796 \\
\hline 57 & Lautaro .................................. & 217.743 \\
\hline 58 & Santa Luisa & 558.633 \\
\hline \multicolumn{2}{|l|}{ Total } & 14.403 .915 \\
\hline
\end{tabular}

Tabla 5. Elaboración anual habida en las oficinas salitreras en 1899 (Campaña 1900: 79).
En la Tabla 3 señalábamos con claridad que Antofagasta, que expresa al cantón Bolivia o Central, era hacia fines del s. XIX escasamente relevante en el marco general de la producción y exportación de nitrato, comparado no solo con Tarapacá sino también con Tocopilla (cantón El Toco). Por eso, no sorprende la aseveración de los ingenieros alemanes Semper y Michels, quienes realizaron un exhaustivo estudio de la economía salitrera en 1904:

"...de los cinco distritos productores de salitre, Antofagasta es el menos importante. La única oficina que trabaja en este distrito, elabora en el puerto de Antofagasta un caliche muy pobre, rico en sal y sulfatos, que es traído a la costa por el ferrocarril de Antofagasta a Oruro, desde Pampa Alta, Carmen Alto, Pampa Central y Salar del Carmen. Los yacimientos están como a 80 kilómetros de la costa, en ambas laderas del valle de Sierra Gorda, cruzado por la vía férrea. Cerca de esas pampas, la firma Carrasco, Zanelliy $C^{a}$ ha levantado una nueva oficina" (Semper y Michels 1908:23).

No imaginaron los sabios alemanes que muy pronto, cuando se dio a conocer su obra en Chile -como lo aseveran los propios traductores chilenos, el ingeniero Javier Gandarillas y el abogado Orlado Ghigliotto- ya habían surgido varias sociedades para explotar las pampas del distrito Antofagasta o cantón Bolivia. Esas sociedades eran Progreso, Carmen, Candelaria, Leonor, Riviera, Loa, Araucana, Anita, Aurelia, Celia. La salitrera a la que hacían referencia como "la única que trabaja en este distrito" era aquella que organizó la Compañía de Salitres y Ferrocarril de Antofagasta (C.S.F.A.), heredera de la famosa Sociedad Exploradora del Desierto de Atacama, creada por José Santos Ossa, Francisco Puelma y Manuel Antonio Lama. Años después surgirían dos salitreras con los nombres de Ossa y Puelma, perpetuándolos en la historia del salitre del cantón Bolivia. La salitrera de la firma Carrasco, Zanelli y $\mathrm{C}^{\mathrm{a}}$ que mencionan Semper y Michels es Lastenia, que tuvo una larga vida como oficina, ubicada en Salinas, en la proximidades de Chacabuco.

Bermúdez (1984:121) respecto de la explotación del nitrato, destaca la "prioridad de la provincia salitrera de Tarapacá para descartar la porfiada insistencia de muchos de que Antofagasta fue la cuna de esta industria". La pregunta que cabe hacerse aquí es si dicha prioridad lo fue también durante el ciclo de expansión del nitrato o, si por el contrario, otro distrito salitrero tomó el liderazgo que tuvo Tarapacá durante el largo período anterior a la Guerra del Pacífico. 
1. Riviera. Carmen Alto. Representada por ella misma, con domicilio en Valparaíso, embarcaba por Antofagasta.

2. Carmen Alto. Carmen Alto. De la $C^{a}$ de Salitres de Antofagasta, representada por ella misma, con domicilio en Valparaíso y Gerencia en Antofagasta, embarcaba por este puerto.

3. Florencia. Carmen Alto. De The Florencia Nitrate $C^{0}$ Ltd., representada por Clarke, Bennett y $C^{a}$, con domicilio en Iquique y Agencia en Antofagasta, embarcaba por Mejillones.

4. Celia. Carmen Alto. De The Pacific Nitrate $C^{\circ}$ Ltd., representada por Harrington, Morrison y $C^{a}$, con domicilio en Iquique y Agencia en Antofagasta, embarcaba por Mejillones.

5. Aurelia. Estación Salinas. De The Pacific Nitrate $C^{0}$ Ltd., representada por Harrington, Morrison y $C^{a}$, con domicilio en Iquique y Agencia en Antofagasta, embarcaba por Mejillones.

6. Lastenia. Estación Salinas. De la Ca Salitrera Lastenia, representada por ella misma, con domicilio en Valparaíso y Agencia en Antofagasta, embarcaba por este puerto.

7. Carmela. Estación Salinas. De The Fortuna Nitrate $C^{\circ}$ Ld., representada por Harrington, Morrison y $C^{a}$, con domicilio en Iquique y Agencia en Antofagasta, embarcaba por Mejillones.

8. Agustín Edwards. Pampa Central. De la $C^{a}$ de Salitres de Antofagasta, representada por ella misma, con domicilio en Valparaíso y Gerencia en Antofagasta, embarcaba por este puerto. En proceso de construcción, se espera que elabore a partir de marzo 1909.

9. Ausonia. Pampa Central. De la $C^{a}$ Salitrera Progreso de Antofagasta, representada por ella misma, con domicilio en Santiago y gerencia en Antofagasta, embarcaba por este puerto.

10. Leonor. Pampa Central. De Leonor Nitrate $C^{\circ}$ Ltd., representada por Clarke, Bennett y $C^{a}$, con domicilio en Iquique y Agencia en Antofagasta, embarcaba por Mejillones.

11. Anita. Pampa Central. De la Ca Salitrera Pampa Alta, representada por ella misma, con domicilio en Valparaíso y Agencia en Antofagasta, embarcaba por este puerto.

12. Candelaria. Pampa Central. De C Salitrera Candelaria, representada por ella misma con domicilio en Valparaíso y Agencia en Antofagasta, embarcaba por Mejillones.

13. Luisis. Pampa Central. De la $C^{a}$ Salitrera Esmeralda, representada por ella misma con domicilio en Valparaíso y Agencia en Antofagasta, embarcaba por Mejillones.

14. Filomena. Pampa Alta. De la C $C^{a}$ Salitrera Progreso de Antofagasta, representada por ella misma, con domicilio en Santiago y gerencia en Antofagasta, embarcaba por este puerto.

15. Curicó. Pampa Central. De la C ${ }^{\text {a }}$ Salitrera El Loa, representada por ella misma con domicilio en Valparaíso, embarcaba por Mejillones.

16. Aconcagua. Pampa Alta. De la Ca Salitrera Progreso de Antofagasta, representada por ella misma, con domicilio en Santiago y gerencia en Antofagasta, embarcaba por este puerto.

Tabla 6. Oficinas salitreras en funcionamiento en el cantón Bolivia en 1908-1909 (Silva Narro 1909: 264-273).

En particular nos preguntamos si ese liderazgo pudo ser tomado por el distrito de Antofagasta, el cantón Bolivia, o la sumatoria de éste, El Toco y Aguas Blancas, para considerar a la provincia de Antofagasta en su totalidad.

El ciclo de expansión del nitrato llegaba a su punto máximo hacia 1908-09. En la Tabla 6 se detallan las oficinas salitreras que estaban en operaciones. Para entonces este cantón ya parecía consolidado y su producción comenzaba a tener una importancia significativa en las estadísticas salitreras, superando a sus vecinas del sur, Pampa Blanca y Taltal, y siendo superada por El Toco y por cierto Tarapacá, que seguía liderando la producción y exportación de nitrato y yodo (Tabla 7; ver también Tabla 1).

Esta desagregación del salitre exportado por puertos de embarque nos permite observar mejor la capacidad productiva por cantones o zonas salitreras, pues los puertos de Pisagua, Junín y Caleta Buena exportaban la producción de los cantones de la zona norte de Ta- 


\begin{tabular}{|l|c|c|}
\hline \multicolumn{1}{|c|}{ Puertos } & Cantidades exportadas & $\%$ \\
\hline Iquique & $13.285,85$ & 26.3 \\
\hline Caleta buena & $5.837,57$ & 11.5 \\
\hline Junín & $1.783,56$ & 3.5 \\
\hline Pisagua & $2.482,41$ & 4.9 \\
\hline Tocopilla & $6.409,95$ & 12.6 \\
\hline Mejillones & $5.589,11$ & 11.0 \\
\hline Antofagasta & $6.163,22$ & 12.2 \\
\hline Caleta Coloso & $3.266,41$ & 6.4 \\
\hline Taltal & $5.963,22$ & 11.8 \\
\hline Total general & $50.481,33$ & 100 \\
\hline
\end{tabular}

Tabla 7. Salitre exportado en 1910 por los distintos puertos, expresado en quintales españoles (Cariola y Sunkel 1991: 133).

rapacá (Zapiga, Dolores, Catalina, Negreiros y Huara). El puerto de Iquique la de las zonas centro y sur de la provincia desde el cantón La Peña hasta el de Lagunas. Tocopilla, como sabemos, representaba la producción del cantón El Toco, y los puertos de Mejillones y Antofagasta la del cantón Bolivia o Central (una proporción también del cantón Aguas Blancas). La caleta Coloso exportaba el salitre de los cantones El Boquete y Aguas Blancas. El puerto de Taltal hacía lo propio con el nitrato del cantón de su mismo nombre.

Vemos que los puertos de Mejillones y Antofagasta alcanzaban ya el $23.2 \%$ del total de exportaciones, comparado con Tarapacá que llagaba al 46.2\%, Tocopilla al 12.6\%, Coloso al $6.4 \%$ y Taltal al 11.8\%. Es decir, Antofagasta, de ser el último distrito salitrero en importancia, ocupaba ahora el segundo lugar después de Tarapacá en apenas una década. Incluso Antofagasta, tomada en su conjunto como provincia -según un registro de la comisión parlamentaria encargada de estudiar las necesidades de las provincias de Tarapacá y Antofagasta en 1913-, logró una producción total de 31 millones y medio de quintales de salitre mientras Tarapacá alcanzó 23 millones y medio. La producción de yodo por su parte fue de $389.665 \mathrm{k}$ y se repartió en partes aproximadamente iguales entre ambas provincias (Cámara de Diputados 1913: 186). Esto a pesar de que, en dicho año, había 99 salitreras en Tarapacá, 10 en Tocopilla, 25 en Antofagasta, 14 en Aguas Blancas y 18 en Taltal. La salitrera que alcanzó la mayor producción individual fue Alianza del cantón Buenaventura de Tarapacá, y la que tuvo la mayor producción de yodo fue Prosperidad, del cantón El Toco. Podemos observar que la provincia de Antofagasta comenzaba a alcanzar al tradicional líder en la producción y exportación de salitre y yodo: Tarapacá.

Otro factor que llegaría a favorecer al distrito de Antofagasta, específicamente al cantón Bolivia o Central a partir de 1914, fue el estallido de la Primera Guerra Mundial, porque las oficinas salitreras alemanas estuvieron impedidas de seguir exportando nitrato, especialmente por el uso bélico que podía dársele a esta sustancia. Las empresas alemanas fueron comercialmente aisladas desde un inicio del conflicto e incluso el Reino Unido presionó a las firmas inglesas para que despidieran a su personal alemán (Couyoumdjian 1986: 141). Los barcos de la línea "P"10 quedaron anclados en los puertos de embarque esperando el término de la guerra. Esta medida afectó directamente a los cantones El Toco y Taltal, donde se ubicaba el mayor número de salitreras de capitales de esa nacionalidad.

\footnotetext{
${ }^{10}$ Para una mejor comprensión de las líneas de veleros salitreros ver Lubbock (2003).
} 


\begin{tabular}{|c|c|c|c|}
\hline \multirow{2}{*}{ Oficinas } & \multicolumn{3}{|c|}{ Desde el $1^{\circ}$ de julio al 31 de agosto } \\
\hline & $1918-19$ & $1917-18$ & $1916-17$ \\
\hline Tarapacá...... & 5.662 .495 & 4.918 .568 & 4.980 .214 \\
\hline Tocopilla...... & 411.083 & 1.212 .151 & 1.302 .842 \\
\hline Antofagasta................... & 4.666 .832 & 3.588 .702 & 2.783 .915 \\
\hline Aguas Blancas................ & 850.099 & 845.166 & 333.477 \\
\hline Taltal.......................... & 1.041 .900 & 1.038 .503 & 619.999 \\
\hline Total & 12.632 .409 & 11.603 .090 & 10.020 .447 \\
\hline
\end{tabular}

Tabla 8. Demostración comparada. Salitre exportado en quintales españoles en las distintas regiones salitreras (Asociación Salitrera de Propaganda).

El auge alcanzaba por entonces también al ferrocarril de Antofagasta a Bolivia, considerado por The Time, "con la posible excepción de la Peruvian Corporation, la empresa industrial más importante en la costa del Pacífico" (Blakemore 1996:102).

Mientras en Tarapacá dominó el capital inglés, en el cantón Bolivia o Central prevaleció el capital nacional, como se puede analizar de las compañías salitreras que construyeron las principales oficinas: $\mathrm{C}^{\mathrm{a}}$ de Salitres de Antofagasta, The Florencia Nitrate $C^{\circ}$ Ltd., The Pacific Nitrate $C^{\circ}$ Ltd., $C^{a}$ Salitrera Lastenia, The Fortuna Nitrate $C^{0} L_{t d}$., $C^{a}$ Salitrera Progreso de Antofagasta, Leonor Nitrate $C^{\circ} \mathrm{Ltd}$., $C^{a}$ Salitrera Pampa Alta, C ${ }^{a}$ Salitrera Candelaria, Ca Sa-

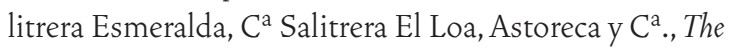
Amelia Nitrate $\mathrm{C}^{\circ}$ Ltd., Carrasco, Zanelli, Baburizza, Bruna y $\mathrm{C}^{\circ}, \mathrm{C}^{\mathrm{a}}$ Salitrera Cerrillos, Baburizza, Lukinovic y $\mathrm{C}^{\mathrm{o}}$, Jorge Sabioncello Ch., C ${ }^{\text {a }}$ Salitrera Perseverancia (Silva Narro 1909, 1913, 1917; Ffrench-Davis y Rauld 1923).

En la Tabla 8, puede apreciarse el comportamiento del cantón Bolivia o Central al término de la Primera Guerra Mundial. Se puede observar con claridad el crecimiento del distrito salitrero de Antofagasta entre los años 1916 y 1919 en comparación con los otros cuatro, donde Tarapacá sigue dominando la exportación de nitrato en términos absolutos. Pero, proporcionalmente su importancia ha comenzado a caer significativamente en beneficio de otros distritos, especialmente el de Antofagasta. El comportamiento del cantón Bolivia o Central en las décadas siguientes a 1920 amerita un estudio específico, pues definitivamente Tarapacá como provincia salitrera pierde el liderazgo ante la provincia de Antofagasta, es- pecialmente por la introducción de un nuevo sistema de lixiviación del nitrato de origen norteamericano, llamado Guggenheim, superando al sistema Shanks, de origen inglés, que fue el principal motor de la expansión salitrera hasta 1920 . Entre 1921 y enero de 1923 , se publicaron las patentes sobre este nuevo procedimiento de extracción y elaboración del nitrato de sodio, que fueron concedidas a los señores Guggenheim Bros. ${ }^{11}$

En definitiva, de la solitaria oficina salitrera que registraron en 1904 los ingenieros alemanes Semper y Michels, a las 16 oficinas que estaban elaborando en 1909 y las 25 que observamos en 1919, el cambio es manifiesto (Tabla 9). Mientras las ricas pampas calicheras de Tarapacá, especialmente de las zonas central y norte, comenzaban a agotarse, en Antofagasta se iniciaba un nuevo ciclo salitrero que estaba en expansión. Ya no era el mismo mercado internacional del nitrato donde las combinaciones salitreras pudieron especular con el precio hasta terminar agotando la paciencia de los consumidores, especialmente de Alemania. Según Enrique Reyes Navarro, las combinaciones, que eran organizadas por las grandes compañías salitreras con sede en Londres (Permanent Nitrate Committee) y en Iquique y Valparaíso (Asociación Salitrera de Propaganda):

“...optaron por la solución más fácil: un juego que conducía inevitablemente a la alza y baja del producto, trayendo como consecuencia en la mediana y larga duración una pérdida de mercado. Es interesante notar que una serie de nuevos mercados se abrirá

${ }^{11}$ En varios números de la revista Caliche se analizan diferentes métodos de extracción y lixiviación del nitrato, entre ellos el sistema Guggenheim (Caliche 4, año V, 1923: 151). 


\begin{tabular}{|c|c|c|c|}
\hline \multicolumn{4}{|c|}{ Cantón Bolivia, 1919} \\
\hline Salitrera & Ubicación & Compañía salitrera & Puerto embarque \\
\hline 1. Aconcagua & Paradero La Noria & Baburizza, Lukinovic y $\mathrm{C}^{\circ}$ & Mejillones \\
\hline 2. Agustín Edwards & Central & $\mathrm{C}^{\mathrm{a}}$ de Salitres de Antofagasta & Antofagasta \\
\hline 3. Angamos & Estación Unión & $\mathrm{C}^{\text {a Salitrera El Loa }}$ & Mejillones \\
\hline 4. Aníbal Pinto & Paradero Maipú & $\mathrm{C}^{\mathrm{a}}$ de Salitres de Antofagasta & Antofagasta \\
\hline 5. Anita & Estación Unión & $\mathrm{C}^{\text {a Salitrera El Loa }}$ & Mejillones \\
\hline 6. Araucana & Estación Unión & $C^{a}$ Salitrera Lastenia & Antofagasta y Mejillones \\
\hline 7. Aurelia & Paradero Salinas & The Fortuna Nitrate $\mathrm{C}^{\circ} \mathrm{Ltd}$. & Mejillones \\
\hline 8. Blanco Encalada & Salinas & Carrasco y Zanelli & Antofagasta y Mejillones \\
\hline 9. Candelaria & Estación Unión & $C^{a}$ Salitrera El Loa & Mejillones \\
\hline 10. Carlos Condell & Paradero Santa Rosa & $\mathrm{C}^{\mathrm{a}}$ de Salitres de Antofagasta & Antofagasta \\
\hline 11. Carmela & Salinas & The Fortuna Nitrate $C^{\circ}$ Ltd. & Mejillones \\
\hline 12. Cecilia & Paradero Peineta & $\mathrm{C}^{\text {a }}$ Salitrera El Loa & Mejillones \\
\hline 13. Celia & Salinas & The Fortuna Nitrate $C^{\circ}$ Ltd. & Mejillones \\
\hline 14. Curicó & Paradero La Placilla & $C^{a}$ Salitrera El Loa & Mejillones \\
\hline 15. Ercilla & Baquedano Longitudinal & $\mathrm{C}^{\text {a }}$ Salitrera Cerrillos & Mejillones \\
\hline 16. Filomena & Paradero Solitario & Baburizza, Lukinovic y C. & Mejillones \\
\hline 17. Francisco Puelma & Carmen Alto & $\mathrm{C}^{\mathrm{a}}$ de Salitres de Antofagasta & Antofagasta \\
\hline 18. Higinio Astoreca & Zona Longitudinal & Astoreca y $\mathrm{C}^{\mathrm{a}}$ & Mejillones \\
\hline 19. José Santos Ossa & Paradero Ossa & $\mathrm{C}^{\mathrm{a}}$ de Salitres de Antofagasta & Antofagasta \\
\hline 20. Lastenia & Salinas & $C^{a}$ Salitrera Lastenia. & Mejillones \\
\hline 21. Lina & Sierra Gorda & Jorge Sabioncello Ch. & Antofagasta \\
\hline 22. Luisis & Estación Unión & 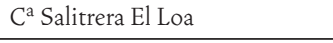 & Mejillones \\
\hline 23. María & Paradero Placilla & $\mathrm{C}^{\text {a }}$ Salitrera El Loa & Mejillones \\
\hline 24. Perseverancia & Paradero Solitario & $C^{a}$ Salitrera Perseverancia & Mejillones \\
\hline 25. Sargento Aldea & Paradero El Buitre & $\mathrm{C}^{\mathrm{a}}$ de Salitres de Antofagasta & Antofagasta \\
\hline
\end{tabular}

Tabla 9. Oficinas que estaban produciendo en el cantón Bolivia o Central cuando deviene la crisis salitrera en 1920 (Silva Narro 1919).

o, algunos nuevos incrementarán su consumo solo después de 1909, cuando la última Combinación fallece de muerte natural" (Reyes Navarro 1985: 194).

Alejandro Bertrand venía denunciando esta especulación desde que asumió su cargo como Inspector Fiscal de Propaganda Salitrera (1910). A su vez, Semper y Michels (1908: 150) señalaron con claridad:

"el predominio de los intereses ingleses en la constitución del directorio de la Asociación Salitrera de Propaganda y de la Com- binación: seis ingleses (con el presidente), dos alemanes (con el vicepresidente), un españoly un italiano".

\section{* Conclusiones}

El cantón Central, Bolivia o El Loa, tuvo ciertas características que lo diferencian respecto de los otros cantones salitreros al norte y sur del paralelo $23^{\circ} \mathrm{S}$, como su temprana explotación por parte de la Compañía de Salitres y Ferrocarril de Antofagasta (C.S.F.A.), cuya ac- 
tividad económica tuvo una directa relación con el conflicto entre Bolivia y Chile que condujo a la Guerra del Pacífico. Fue este cantón el centro del litigio que dio origen a la guerra, a diferencia de los litigios del Toco sobre la propiedad de terrenos salitrales que fueron posteriores a dicho conflicto y que se extendieron por varias décadas (González 2006). Los litigios de terrenos en Tarapacá se produjeron antes y después de la Guerra del Pacífico; primero, producto del intento monopólico del gobierno peruano, y después como resultado de la especulación empresarial inglesa.

A pesar de no ser el cantón más productivo fue el primero cuyo ferrocarril tuvo una proyección internacional, mientras que la provincia salitrera por antonomasia, Tarapacá, no pudo concretar ninguno de los proyectos ferroviarios de conexión con Bolivia desde 1864 en adelante (Castro 2003). De tal modo, la vinculación entre Antofagasta y Oruro permitió aumentar la presencia boliviana en todo el territorio salitrero, especialmente en los cantones Central y El Toco, consolidando la ya significativa población de esa nacionalidad en Tarapacá (González 2009).

Del éxito del sistema Guggenheim en las oficinas María Elena y Pedro de Valdivia -ambas más próximas al que fuera el cantón El Toco- hemos podido recoger importantes esfuerzos tecnológicos que se realizaron en oficinas salitreras del cantón Central, como las experimentaciones realizadas en las oficinas Lastenia y Araucana por parte de los químicos Ludovico Perroni y Carlos Grillo (sistema Grillo-Perroni), así como las realizadas por E. Almeyda Arroyo. Fueron las salitreras del cantón Central las escogidas para ensayar nuevos procedimientos durante la compleja década de 1920 -cuando la crisis se instalaba definitivamente en Chile al término de la Primera Guerra Mundial- con científicos como los ya nombrados además de Manuel Ossa Ruiz, J. Berkwood Hobsbawn, Jules R. Lortsch, Eduardo Tasso, entre otros, sin dejar de destacar la labor educativa e informativa de Belisario Díaz Ossa, director de la revista Caliche.

Uno de los esfuerzos que mejores resultados obtuvieron fueron aquellos de las experimentaciones en la oficina Delaware del cantón Central (posteriormente Alberto Bascuñán) con el procedimiento Allen, que significó el abandono definitivo del sistema Shanks. De tal forma, el cantón Central, a diferencia de los de Tarapacá y Taltal, comenzó a enfrentar la crisis salitrera con más previsión. Es por esto que amerita un estudio más detallado a partir de los años posteriores a la Primera Guerra Mundial y hasta la gran crisis de 1930, porque fue durante esos años cuando sus salitreras tuvieron un papel más significativo en la historia del salitre.

Tarapacá fue la provincia que experimentó en forma más notoria las consecuencias de la falta de previsión del gobierno y de los salitreros respecto al comportamiento futuro del mercado del nitrato. Sin embargo, en Antofagasta, especialmente debido a la construcción de las grandes oficinas donde se instaló el sistema Guggenheim, ${ }^{12}$ todavía el futuro tenía color salitre.

\footnotetext{
${ }^{12}$ Un estudio sobre ambos sistemas de lixiviación (Shanks y Guggenheim) en Antofagasta y sus impactos sociales es el de J. A. González (2003).
}

\section{* Referencias citadas}

ACADEMIA DE CIENCIAS ECONÓMICAS, 1926. Semana del salitre celebrada en Santiago de Chile, abril 1926. Imprenta y Litografía La Ilustración, Santiago.

BERMÚdEZ, O., 1963. Historia del salitre desde sus orígenes hasta la guerra del Pacífico. Ediciones Universidad de Chile, Santiago.

1984. Historia del salitre desde la guerra del Pacífico hasta la revolución de 1891. Ediciones Pampa Desnuda, Santiago.
BERTRAND, A., 1910. La crisis salitrera. Louis Michaud Editor, París.

BILLINGHURST, G., 1886. Estudio sobre la geografía de Tarapacá. Imprenta El Progreso, Santiago.

BLAKEMORE, H., 1977. Gobierno chileno y salitre inglés 1886-1896: Balmaceda y North. Editorial Andrés Bello, Santiago.

1996. Historia del Ferrocarril de Antofagasta a Bolivia 1888-1988. 
Edición Mercedes Gajú-Impresos Universitarios, Santiago.

CARIOLA, C. y O. SUNKEL, 1991. Un siglo de historia económica de Chile 1830-1930. Editorial Universitaria, Santiago.

CÁMARA DE DIPUTADOS, 1913. Comisión Parlamentaria encargada de estudiar las necesidades de las provincias de Tarapacá y Antofagasta. Zig-Zag, Santiago.

CARRASCO, S., 1991. Historia de las relaciones chileno-bolivianas. Editorial Universitaria, Santiago.

CASTRO, L., 2003. Espejismos en el desierto: Proyectos ferroviarios e integración subregional (Tarapacá 1864-1937). Si Somos Americanos vol. 5 (4): 21-48.

COUYOUMDJIAN, J. R., 1986. Chile y Gran Bretaña durante la primera guerra mundial y la posguerra, 1914-1921. Editorial Andrés Bello, Santiago.

FFRENCH-DAVIS y R. y P. RAOULD (Eds.). 1923 Guía general de las provincias de Tarapacá y Antofagasta, 1923. Editorial Lemare y $\mathrm{C}^{\mathrm{a}}$, Iquique.

GONZÁLEZ, J. A., 2003. La pampa salitrera en Antofagasta. Auge y ocaso de una era histórica. La vida cotidiana durante los ciclos Shanks y Guggenheim en el desierto de Atacama. Corporación Pro Antofagasta, Imprenta Ercilla, Antofagasta.

GONZÁLEZ, S., 2002. Hombres y mujeres de la pampa. Tarapacá en el ciclo de expansión del salitre. Ediciones LOM, Santiago.

2006. El Toco y la Guerra del Pacífico. Una hipótesis de trabajo. Hombre y Desierto 13: 7-27.

2009. La presencia boliviana en la sociedad del salitre y la nueva definición de la frontera: Auge y caída de una dinámica transfronteriza (Tarapacá 1880-1930). Chungara 41: 71-81.

HERNÁNDEZ, R., 1930. El salitre. Resumen histórico desde su descubrimiento y explotación. Imprenta Fisher Hnos., Valparaíso.
HOBSBAWN, I. B., 1919. La ciencia y el porvenir de la industria salitrera. Caliche 7: 261-267.

LUBBOCK, B., 1953. The nitrate clippers. Brown, Son \& Ferguson Ltd., Glasgow.

MURAOUR, H., 1922. El nitrógeno en Alemania; antes, durante y después de la guerra. Caliche 6:328-330.

RAVEST, M., 1983. La compañia salitrera y la ocupación de Antofagasta 1878-1879. Editorial Andrés Bello, Santiago.

REYES NAVARRO, E., 1985. El mercado mundial del salitre. Nueva Historia. Revista de Historia de Chile 15-16: 181-214.

SEMPER E. y E. MICHELS, 1908. La industria del salitre en Chile. Imprenta Barcelona, Santiago.

SILVA NARRO, D., 1909. Guía administrativa, industrial y comercial de las provincias de Tacna, Tarapacá y Antofagasta. Imprenta Gutenberg, Santiago.

1913. Guía administrativa, industrial y comercial de las provincias de Tacna, Tarapacá y Antofagasta. Imprenta Gutenberg, Santiago.

1917. Guía administrativa, industrial y comercial de las provincias de Tacna, Tarapacá y Antofagasta. Imprenta Gutenberg, Santiago.

1919. Guía administrativa, industrial y comercial de las provincias de Tacna, Tarapacá y Antofagasta. Imprenta Gutenberg, Santiago.

STEENHUIS, A., 2003. Windjammers in Delfzijl. Godert Walter, Amsterdam.

THOMPSON, I. y D. ANGERSTEIN, 1997. Historia del ferrocarril en Chile. Dirección de Bibliotecas Archivos y Museos, Centro de Investigaciones Diego Barros Arana, Santiago.

VIDAL, J. 1953. Veinte años después. La tragedia del salitre. Imprenta El Imparcial, Santiago. 